\title{
Summary of the best evidence for the prevention of intraoperative unplanned hypothermia in patients undergoing laparoscopic surgery
}

\author{
Mindan Wang ${ }^{1}$, Yingying Jiang ${ }^{1}$, Wenjuan Han ${ }^{1}$, Ling Jiang ${ }^{2}$, Chenrong Mao ${ }^{2}$ \\ ${ }^{1}$ Operating Room, The Affiliated Suzhou Hospital of Nanjing Medical University, Suzhou Municipal Hospital, Suzhou, China; ${ }^{2}$ Nursing \\ Department, The Affiliated Suzhou Hospital of Nanjing Medical University, Suzhou Municipal Hospital, Suzhou, China \\ Contributions: (I) Conception and design: C Mao; (II) Administrative support: M Wang; (III) Provision of study materials or patients: Y Jiang; (IV) \\ Collection and assembly of data: W Han; (V) Data analysis and interpretation: L Jiang; (VI) Manuscript writing: All authors; (VII) Final approval of \\ manuscript: All authors. \\ Correspondence to: Chenrong Mao. Nursing Department, The Affiliated Suzhou Hospital of Nanjing Medical University, Suzhou Municipal Hospital, \\ Suzhou, China. Email: 2529559092@qq.com.
}

\begin{abstract}
Background: This study aimed to search, analyze, and summarize relevant evidence for the prevention of unplanned hypothermia in patients undergoing laparoscopic surgery, so as to reduce the incidence of unplanned hypothermia in patients undergoing laparoscopic surgery and provide a reference for clinical medical staff.

Methods: According to the evidence pyramid model, relevant literature were retrieved by computer in databases. Literature quality evaluation and the evidence grading system of the Australian JBI EvidenceBased Health Care Centre Evidence Recommendation Rating System were used to evaluate the literature quality and determine the level of evidence. The time limit for retrieval was from the establishment of the databases to March 31, 2021.
\end{abstract}

Results: A total of 12 studies were included in this study. Of these, 5 evidence items were finally extracted, and 15 pieces of best evidence were summarized, including the risk assessment of hypothermia in patients undergoing laparoscopic surgery, temperature monitoring, ambient temperature, passive insulation measures, and active insulation measures.

Conclusions: The evidence for the prevention of unplanned hypothermia in patients undergoing laparoscopic surgery provided evidence-based approaches for reducing the incidence of intraoperative hypothermia for clinical staffs. It is suggested that the cultural characteristics of China, medical resources, and patients' own conditions should be considered when applying the evidence.

Keywords: Laparoscopic; unplanned hypothermia; intraoperative; evidence-based nursing

Submitted Jul 16, 2021. Accepted for publication Sep 15, 2021.

doi: $10.21037 / g s-21-578$

View this article at: https://dx.doi.org/10.21037/gs-21-578

\section{Introduction}

Laparoscopic technology has the advantages of small trauma, quick recovery, fewer complications, and wide indications (1), which can significantly relieve patients' physical pain and promote postoperative recovery. Therefore, it is widely used in clinical practice. However, under the influence of general anesthesia, $\mathrm{CO}_{2}$ pneumoperitoneum, and other factors, unplanned hypothermia can easily occur in patients undergoing laparoscopic surgery (2). Hypothermia refers to core body temperature $<36^{\circ} \mathrm{C}$, and the incidence of hypothermia in laparoscopic surgery is as high as $50-90 \%$ (3). Hypothermia can increase many perioperative complications, like intraoperative coagulation dysfunction, high intraoperative blood loss, prolonged 
postoperative recovery time, postoperative ileus, leakage, pain, shivering, increased incidence of surgical site infection and cardiovascular disease. In severe cases, it can cause ventricular fibrillation, cardiac arrest, and even death $(4,5)$. In laparoscopic operations, the operating space is limited by the limited space in the abdominal cavity. Therefore, gas is used to create an artificial pneumoperitoneum, thereby increasing the operating space in the abdominal cavity. $\mathrm{CO}_{2}$ is a commonly used gas in laparoscopic surgery, because it is an inert gas that does not support or burn and does not cause smog. $\mathrm{CO}_{2}$ is highly soluble in the blood, so it can be absorbed by the body and discharged from the lungs without leaving a legacy. The core and skin temperature are higher in warm and humidified $\mathrm{CO}_{2}(6)$. The Asia Pacific Society of Infection Control (APSIC) guidelines for the prevention of surgical site infections [2019] emphasizes that hypothermia is an important factor affecting the occurrence of surgical site infection (7). Previous studies have been focused on the topic of hypothermia during laparoscopic surgery. However, the conclusion of these studies was varied and scattered. A summary of the best evidence for the prevention of intraoperative unplanned hypothermia in patients undergoing laparoscopic surgery is urgent to guide clinical work. Therefore, this study summarized the best evidence on intervention measures for the prevention of unplanned hypothermia during laparoscopic surgery, so as to reduce the incidence of unplanned hypothermia during laparoscopic surgery and provide a reference for clinical medical staff.

\section{Methods}

\section{Question identification}

The question of this study was to determine which measures could effectively prevent intraoperative unplanned hypothermia in patients undergoing laparoscopic surgery. To obtain the best evidence, the PICOT approach was used to form the initial questions for this evidence-based care, namely, $\mathrm{P}$ (population): age $\geq 18$ years, under general anesthesia, and laparoscopic surgery; I (intervention): intervention strategies for intraoperative unplanned hypothermia; C (comparison): current clinical nursing measures; $\mathrm{O}$ (outcome): prevention/reduction of unplanned hypothermia; $\mathrm{T}$ (types of evidence): guidelines, summary of evidence, expert consensus, systematic review, metaanalysis, randomized controlled trial.

\section{Retrieval strategy}

According to the " $6 \mathrm{~S}$ " evidence model (8), computer evidence retrieval was carried out from the establishment of the databases to March 31, 2021. The databases were British Medical Journal (BMJ) Best Practice, UpToDate, Cochrane Library, JBI International Collaborating Centre Library for Evidence-Based Health Care, Guidelines International Network (GIN), National Institute for Health and Care Excellence (NICE), Scottish Intercollegiate Guidelines Network (SIGN), Medline, PubMed, Embase, Web of Science, CNKI, Wanfang Database, CQVIP. The Chinese search keywords were "Laparoscopic/minimally invasive/endoscope", "Perioperative/perioperative period/ intraoperative", "hypothermia/hypothermy". The English search words were "laparoscope/laparoscopic/minimal invasive/endoscope/endoscopy", "perioperative/perioperative/intra-operative", and "hypothermia".

\section{Literature inclusion and exclusion criteria}

The literature inclusion criteria were as follows: the subjects were patients aged $\geq 18$ years who underwent general anesthesia; the contents of the study were related to perioperative hypothermia; the types of literature included guidelines, summary of evidence, expert consensus, systematic evaluation, and randomized controlled trial; the language of publications was limited to Chinese or English; for revised or updated guidelines, the latest edition was included. The literature exclusion criteria were as follows: incomplete information, conference report, abstract only, and literature with low quality evaluation.

\section{Criteria for literature quality evaluation}

According to the literature types, the corresponding evaluation criteria were selected for quality evaluation. The quality evaluation criteria for guidelines were according to the Appraisal of Guidelines for Research and Evaluation Instrument (AGREE II) (9). The evaluation of evidence summaries was carried out using the CASE Evaluation Checklist (10). The included expert consensus was evaluated by the JBI Expert Consensus Quality Evaluation Tool (2016 Edition) (11), which included 6 items, and the evaluation criteria for each item were "yes, no, unclear, and inapplicable". The Assessment of Multiple Systematic Reviews (AMSTAR) was used for systematic review 
evaluation (12). The authenticity of randomized controlled trials was evaluated using the Australian JBI Evidence-based Health Care Centre RCT Authenticity Quality Assessment Tool [2016] (13), and the risk of bias of randomized controlled trials was evaluated using the Cochrane Bias Risk Assessment Checklist [2011] (14).

\section{Evidence quality evaluation}

The quality evaluation of the guidelines was independently completed by 3 investigators who received systematically evidence-based training, and the quality evaluation of the other included literature was independently completed by 2 researchers. Conflicts of evaluation opinions between the 2 researchers were resolved through discussion, or a third investigator evaluated the literature and decided. When evidence conclusions from different sources were repeated or conflicting, the inclusion principle followed in this study was that evidence-based and high-quality evidence was preferred.

\section{Results}

\section{General characteristics of the included literature}

After preliminary retrieval, a total of 48 relevant studies were obtained. After the deletion of duplicates and the exclusion of literature that did not meet the inclusion criteria, 12 studies were finally included, including 1 guideline, 2 evidence summaries, 1 expert consensus, 6 systematic reviews, and 2 randomized controlled trials (Table 1).

\section{Quality evaluation results of the included studies}

Quality evaluation results of the guideline

One guideline (15) was included in this study, and the percentage of standardization in each field is shown in Table 2. The study design was complete and the overall quality was high.

\section{Quality evaluation results of evidence summaries}

In this study, 2 evidence summaries from the JBI $(16,17)$ were included. The CASE Evaluation Checklist (10) was used for literature quality evaluation. Except for the evaluation results of items 3 , 4, and 9, which were "no", and item 8 , which was "partial yes", all the other evaluation results were "yes". The study designs were complete and the overall quality was high. Thus, the 2 evidence summaries were included.

\section{Quality evaluation results of expert consensus}

One expert consensus was included in this study (18), which was from the Wanfang database. Except for item 6, the evaluation results of the other items were all "yes". The study design was complete and the overall quality was high, therefore it was included.

\section{Quality evaluation results of systematic reviews}

In this study, 6 systematic reviews were included, including 3 from the Cochrane Library (19-21), 2 from PubMed $(22,23)$, and 1 from CNKI (24). Among them, the evaluation results of all items in the study of Birch et al. (19) were "yes". In the study of Madrid et al. (20), the evaluation results of items 4 and 5 were "unclear", while the evaluation results of other items were "yes". In the study of Warttig et al. (21), the evaluation result of item 15 was "no", and the evaluation results of other items were "yes". In the study of Nieh et al. (22), the evaluation results of items 4 and 16 were "partially yes", and the evaluation results of other items were all "yes". In the study of Dean et al. (23), the evaluation results of items 5, 6, and 16 were "partially yes", while the evaluation results of other items were "yes". In the study of Wang et al. (24), the evaluation results of items $1,4,10$, and 16 were "partially yes", and the evaluation results of other items were "yes". The study designs were complete, the overall quality was high, and these studies were approved for inclusion.

\section{Quality evaluation results of randomized controlled trials}

A total of 2 randomized controlled trials were included in this study $(25,26)$, both from PubMed. The Australian JBI Evidence-based Health Care Centre RCT Authenticity Quality Assessment Tool [2016] (13) and the Cochrane Bias Risk Assessment Checklist (14) were used for evaluation. In the study by Hara et al. (25), the evaluation result of item 6 "whether results were blinded to the evaluator" was "not clear", while the other items were "yes". In the bias risk assessment, all the items were "low risk", except for the "unclear" evaluation result of item 4 which was "whether results were blinded to the evaluator". In the study by Min et al. (26), except for the evaluation result of item 8 "is follow-up complete? If not, are measures taken to deal with loss of follow-up?" which was "not applicable", the results of other items were "yes". The bias risk assessment was "low risk", except for the evaluation result of "whether results were blinded to the evaluator" in item 4, which was "unclear". The study designs were complete, the overall 
Table 1 Characteristics of included studies

\begin{tabular}{|c|c|c|c|c|}
\hline $\begin{array}{l}\text { Literature } \\
\text { source }\end{array}$ & Author & $\begin{array}{l}\text { Publication } \\
\text { time }\end{array}$ & Literature content/theme & Evidence type \\
\hline Guideline & NICE (15) & 2016 & Management of perioperative body temperature in adults & Guideline \\
\hline JBI & $\begin{array}{l}\text { Sivapuram } \\
\text { et al. (16) }\end{array}$ & 2020 & $\begin{array}{l}\text { The best evidence for the use of active heating to prevent } \\
\text { perioperative hypothermia in patients undergoing major surgery } \\
\text { under general anesthesia }\end{array}$ & Evidence summary \\
\hline Wanfang & Ma et al. (18) & 2017 & $\begin{array}{l}\text { Expert consensus on the prevention and treatment of perioperative } \\
\text { patients with hypothermia }\end{array}$ & Expert consensus \\
\hline Cochrane & Birch et al. (19) & 2016 & $\begin{array}{l}\text { Influence of heat preservation, humidification, or no humidification in } \\
\text { laparoscopic abdominal surgery }\end{array}$ & Systematic review \\
\hline PubMed & Nieh et al. (22) & 2016 & $\begin{array}{l}\text { Effect of forced air heating on the prevention of perioperative } \\
\text { hypothermia }\end{array}$ & Systematic review \\
\hline PubMed & Dean et al. (23) & 2017 & $\begin{array}{l}\text { Heated and humidified carbon dioxide injection is beneficial to } \\
\text { intraoperative core temperature maintenance in laparoscopic surgery }\end{array}$ & Systematic review \\
\hline CNKI & Wang et al. (24) & 2017 & $\begin{array}{l}\text { Meta-analysis of the intraoperative and postoperative effects } \\
\text { of carbon dioxide temperature and humidity in laparoscopic } \\
\text { gynecological surgery }\end{array}$ & Systematic review \\
\hline PubMed & Hara et al. (25) & 2021 & $\begin{array}{l}\text { During endoscopic surgery under general anesthesia, the lithotomy } \\
\text { pad has a higher heating effect than the upper body pad }\end{array}$ & RCT \\
\hline
\end{tabular}

Table 2 Methodological quality evaluation of the guideline

\begin{tabular}{lc}
\hline Variables & Outcome \\
\hline Included literature & NICE \\
Percentage of standardization by field (\%) & 100 \\
Scope and purpose & 87.05 \\
Involved personnel & 96.03 \\
Strictness in the development of the guideline & 100 \\
Explicitness of presentation of the guideline & 79.63 \\
Applicability of the guideline & 100 \\
Independent compilation of the guideline & 6 \\
$\geq 60 \%$ number of fields (number) & 6 \\
$\geq 30 \%$ number of fields (number) & $\mathrm{A}$ \\
\hline
\end{tabular}

quality was high, and these studies were approved for inclusion.

\section{Evidence summary and description}

The contents of the finally included studies were extracted, and 15 pieces of relevant evidence were obtained, as shown in Table 3. All included evidence was graded using the Australian JBI Evidence-Based Health Care Centre Evidence Recommendation Rating System (2014 Edition) (27). The evidence derived from evidence summaries was traced back to its reference in the original literature for the grade division of evidence. According to the validity, feasibility, suitability, and clinical significance of the evidence, the recommendation level of the evidence was determined as grade A or grade B based on the JBI recommendation grading. 
Table 3 Evidence summary for the prevention of intraoperative unplanned hypothermia in patients undergoing laparoscopic surgery

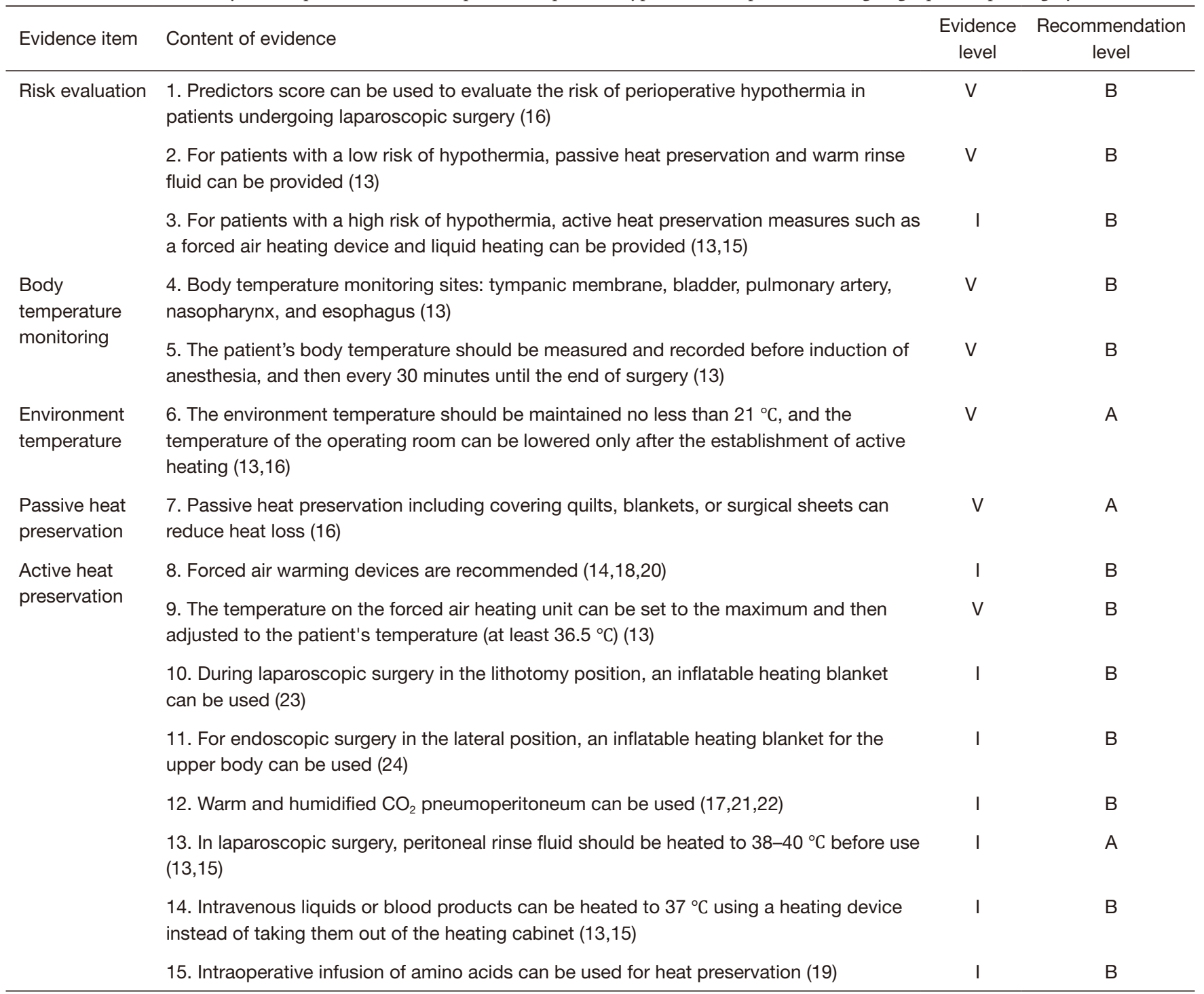

\section{Discussion}

Since its invention, laparoscopic surgery has played an important role in the diagnosis and treatment of abdominal disease because of its characteristics of small surgical trauma, rapid recovery of patients, short hospital stays, good abdominal beauty effect and low surgical cost. Many diseases, like diseases of the biliary system, the spleen, the pancreas, gastrointestinal surgery, the chest, the thyroid, the breast and the gynecology, could be treated by laparoscopic surgery. However, there are some contraindications of laparoscopic surgery, like (I) severe heart, lung, liver, and renal insufficiency; (II) hypervolume lump; (III) Abdominal or diaphragmatic hernia; (IV) Diffuse peritonitis with ileus; (V) Intraperitoneal adhesions were very serious. By summarizing the evidence on the prevention of unplanned hypothermia during laparoscopic surgery, we synthesized evidence from 5 aspects of hypothermia risk assessment, temperature monitoring, environmental temperature, passive insulation, and active insulation, and obtained 15 pieces of best evidence (Table 3).

\section{Risk evaluation}

There is no study compared the incidence difference of unplanned hypothermia in different diseases treated 
by laparoscopic surgery. These are some risk factors of unplanned hypothermia, including age, BMI, ASA level, basic body temperature, complication, surgery time, level type, intraoperative irrigation, time, method and drug of anesthesia, intraoperative infusion and blood transfusion, and operating room temperature (28). Some reported strategies could reduce the risk of unplanned hypothermia, like reducing the surgical time, minimally invasive surgery is the best choice, heating the intraoperative irrigation and infusion fluid, reducing anesthesia time and increasing the environment temperature (28). The first evidence came from the expert consensus on the prevention and treatment of perioperative hypothermia reported by Ma et al. (18). In the preoperative evaluation of hypothermia prevention and treatment, the importance of risk assessment of hypothermia in patients was highlighted, and medical personnel should record the real-time temperature of patients in preoperative evaluation. For the preoperative assessment of patients under general anesthesia, the Probability Scale of Perioperative Hypothermia (also known as the Predictors Score) was recommended (29). This scale is a prediction model of hypothermia established based on the epidemiological results of perioperative hypothermia in patients under general anesthesia in China. The risk probability of intraoperative hypothermia can be obtained by inputting patient-related parameters.

The second and third pieces of evidence were derived from the NICE guidelines (15) and the JBI summary of evidence on the prevention and management of perioperative hypothermia (17). The NICE guidelines (15) indicate that for patients with a low risk of hypothermia, the best strategy is heated fluid, which can provide passive heat preservation, and warm rinse fluid. For patients with a high risk of unplanned hypothermia, the most effective prevention method during 60 and 120 minutes of anesthesia is warm fluid and active warming measures. Active heat preservation measures such as forced air heating devices and heated fluid can be provided. Forced air heating is an efficient way to increase the warming efficacy. The application of forced air heating could also reduce the incidence of complications (30). The JBI summary of evidence on the prevention and management of perioperative hypothermia (17) recommends that patients should be evaluated preoperatively for the risk of unplanned hypothermia. Patients who are assessed as having an increased risk of hypothermia should be treated with a forced air heating device during the operation, even if the anesthetic duration is less than 30 minutes. If it is assessed as high risk, the body temperature should be actively handled. If the preoperative temperature is below $36{ }^{\circ} \mathrm{C}$, forced air warming should be initiated and maintained throughout the operation.

\section{Body temperature monitoring}

Evidence 4 and 5 came from the NICE guidelines (15), which state that body temperature should be measured in one of the following parts: the tympanic membrane, bladder, pulmonary artery, nasopharynx, and esophagus. Measurements of the temporal artery, rectum, and mouth should be considered as indirect results. Temperature measurements of the skin or armpit are excluded as these areas are superficial. Measurements should be taken before induction of anesthesia and every 30 minutes until the end of surgery.

\section{Environment temperature}

The 6th piece of evidence shows the influence of environment temperature on hypothermia. The NICE guidelines (15) and expert consensus (18) both point out that after patients enter the operating room, the environment temperature should be maintained at no less than $21^{\circ} \mathrm{C}$, and the operating room temperature can be lowered after the establishment of active heating.

\section{Passive heat preservation}

The 7th piece of evidence comes from an expert consensus (18), which points out that passive heat preservation, including covering quilts, surgical sheets, and thermal blankets, can reduce heat loss by $30 \%$, but this is not enough to prevent hypothermia in patients after anesthesia, and active heat preservation measures are still needed.

\section{Active heat preservation}

The 8th piece of evidence suggests that air-warming devices are recommended for patients at high risk of hypothermia. An evidence summary from the JBI (16) included 19 randomized controlled studies and concluded that the use of forced air heating devices to initiate active heating should be considered to prevent hypothermia in patients undergoing general anesthesia. In the systematic review (20) retrieved from the Cochrane Library, an analysis of 67 randomized controlled trials showed that forced air 
warming reduces the rate of surgical site infection and complications during abdominal surgery compared with no active heat preservation. A meta-analysis (22) from PubMed that included 29 randomized controlled studies concluded that forced air warming is more effective than passive heat preservation and circulating water pads, and that forced air warming also provides better thermal comfort.

The 9th piece of evidence was derived from the NICE guidelines (15) stating that the temperature setting on the forced air heating device should be set to the maximum and then adjusted to at least $36.5^{\circ} \mathrm{C}$ depending on the patient's intraoperative condition.

The 10th piece of evidence was derived from a PubMed randomized controlled trial (25), which concluded that the use of an inflatable heating blanket to control body temperature during laparoscopic surgery under general anesthesia in the lithotomy position can increase and maintain core and peripheral temperature more than an upper body blanket. It can also reduce the incidence of postoperative shivering and shorten the length of postoperative hospital stay. Another study used underbody warming system in laparoscopic gastrointestinal surgery to prevent the occurrence of hypothermia. The blankets are heated by electricity, and the safety of the blankets has been ensured by advanced production technology. Thus, this is a feasible and effective method in keeping the body temperature of the patients (5).

The 11th piece of evidence was derived from a PubMed randomized controlled trial, which indicates that an upper body blanket can transfer heat to a larger body surface area more efficiently than a lower body blanket. Therefore, in thoracoscopic surgery in the lateral decubitus position, an inflatable upper body blanket is more effective than a lower body blanket in preventing intraoperative hypothermia.

The 12th piece of evidence recommended the use of warm and humidified $\mathrm{CO}_{2}$ gas to induce pneumoperitoneum. A systematic review by Dean et al. (23) including 13 randomized controlled studies concluded that compared with cold and dry $\mathrm{CO}_{2}$, heated and humidified $\mathrm{CO}_{2}$ gas during laparoscopic surgery could improve the maintenance of intraoperative body temperature. In a Cochrane systematic review (19), 21 randomized controlled trials were included, demonstrating that heated and humidified pneumoperitoneum could reduce the decrease in intraoperative core body temperature, but there was no significant difference in operative time, hospital stay, recovery time, and postoperative pain. A systematic review from CNKI (24) included 17 randomized controlled trials and reached the same conclusion that heated and humidified $\mathrm{CO}_{2}$ pneumoperitoneum improved intraoperative core temperature and postoperative pain on the day after surgery. Therefore, warm and humidified $\mathrm{CO}_{2}$ pneumoperitoneum is recommended.

The 13th piece of evidence was derived from the NICE guidelines (15) and JBI evidence summary (17). The JBI evidence summary states that warm abdominal irrigation is clearly effective in preventing perioperative hypothermia, and unheated fluids should not be used in patients undergoing prolonged surgery. The NICE guidelines also specify that all intraoperative peritoneal flushing fluid should be heated to $38-40{ }^{\circ} \mathrm{C}$ in an incubator before use.

The 14th piece of evidence was derived from the NICE guidelines (15) and JBI evidence summary (17). The JBI summary of evidence indicates that the risk of perioperative hypothermia is reduced when intravenous and blood products are heated to $37^{\circ} \mathrm{C}$. The NICE guidelines also state that intravenous fluids of $500 \mathrm{~mL}$ or more should be heated using a liquid heater, rather than taken out of a heating cabinet.

The 15th piece of evidence was derived from a systematic review from the Cochrane Library (21). Fourteen randomized and quasi-randomized controlled trials were included in the systematic review. The study showed that the intraoperative body temperature of patients who received intravenous amino acids was $0.5^{\circ} \mathrm{C}$ higher than that of non-users, which was statistically significant at the end of the operation but not significant at other time points. Therefore, it is suggested that intraoperative intravenous infusion of amino acids can be used for heat preservation.

There are 15 pieces of evidences in preventing unplanned hypothermia in surgical patients. The clinical environment and hospital characteristics are various. The risk and probability of unplanned hypothermia are different in patients from different surgical departments. In the surgical department with high incidence of unplanned hypothermia, more anti-hypothermia methods should be taken as routine. In the department with low incidence of unplanned hypothermia, several positive or negative insulation measure to prevent the occurrence of unplanned hypothermia is enough.

\section{Conclusions}

In this study, the quality of evidence was evaluated in strict accordance with the evaluation criteria. This evaluation was combined with the clinical reality and a comprehensive 
consideration of the feasibility of evidence and other factors. However, most of the included evidence came from western literature, and there may be regional and cultural differences between the foreign medical service system and China's domestic environment. It is suggested that the cultural characteristics, medical resources, and patients' wishes should be taken into account in evidence transformation to comprehensively evaluate domestic patients undergoing laparoscopic surgery and formulate nursing practice standards in line with the Chinese cultural reality. In addition, there is a lack of high-quality evidence regarding preoperative and postoperative heat retention times and target temperature in patients undergoing laparoscopic surgery. In the future, studies should be carried out to explore the effects of different preoperative and postoperative heat preservation times and target temperatures on perioperative hypothermia, so as to provide research evidence for the unification of clinical procedures.

\section{Acknowledgments}

Funding: None.

\section{Footnote}

Conflicts of Interest: All authors have completed the ICMJE uniform disclosure form (available at https://dx.doi. org/10.21037/gs-21-578). The authors have no conflicts of interest to declare.

Ethical Statement: The authors are accountable for all aspects of the work in ensuring that questions related to the accuracy or integrity of any part of the work are appropriately investigated and resolved.

Open Access Statement: This is an Open Access article distributed in accordance with the Creative Commons Attribution-NonCommercial-NoDerivs 4.0 International License (CC BY-NC-ND 4.0), which permits the noncommercial replication and distribution of the article with the strict proviso that no changes or edits are made and the original work is properly cited (including links to both the formal publication through the relevant DOI and the license). See: https://creativecommons.org/licenses/by-nc-nd/4.0/.

\section{References}

1. Qin R, Kendrick ML, Wolfgang CL, et al.
International expert consensus on laparoscopic pancreaticoduodenectomy. Hepatobiliary Surg Nutr 2020;9:464-83.

2. Chen HY, Su LJ, Wu HZ, et al. Risk factors for inadvertent intraoperative hypothermia in patients undergoing laparoscopic surgery: A prospective cohort study. PLoS One 2021;16:e257816.

3. Scott AV, Stonemetz JL, Wasey JO, et al. Compliance with Surgical Care Improvement Project for Body Temperature Management (SCIP Inf-10) Is Associated with Improved Clinical Outcomes. Anesthesiology 2015;123:116-25.

4. Guo L. Guide to Operating Room Nursing Practice. Beijing: People's Medical Publishing House, 2020.

5. Pu Y, Cen G, Sun J, et al. Warming with an underbody warming system reduces intraoperative hypothermia in patients undergoing laparoscopic gastrointestinal surgery: a randomized controlled study. Int J Nurs Stud 2014;51:181-9.

6. Garrett C, Steffens D, Ansari N, et al. A phase I, nonrandomized controlled trial demonstrating the novel technique of cytoreductive surgery and hyperthermic intraperitoneal chemotherapy utilizing warm humidified carbon dioxide insufflation. Colorectal Dis 2021;23:1573-8.

7. Ling ML, Apisarnthanarak A, Abbas A, et al. APSIC guidelines for the prevention of surgical site infections. Antimicrob Resist Infect Control 2019;8:174.

8. Dicenso A, Bayley L, Haynes RB. Accessing pre-appraised evidence: fine-tuning the $5 \mathrm{~S}$ model into a $6 \mathrm{~S}$ model. Evid Based Nurs 2009;12:99-101.

9. Brouwers MC, Kho ME, Browman GP, et al. AGREE II: advancing guideline development, reporting and evaluation in health care. CMAJ 2010;182:E839-42.

10. Foster MJ, Shurtz S. Making the Critical Appraisal for Summaries of Evidence (CASE) for evidence-based medicine (EBM): critical appraisal of summaries of evidence. J Med Libr Assoc 2013;101:192-8.

11. The Joanna Briggs Institute. (2017-07-15). (2018-08-29). Available online: https://jbi.global/

12. Xiong J, Chen RX. An Introduction to a Measurement Tool to Assess the Methodological Quality of Systematic Reviews/Meta-analysis: AMSTAR. Chinese Journal of Evidence-Based Medicine 2011;11:1084-9.

13. The Joanna Briggs Institute. Joanna Briggs Institute Reviewers'manual:2016 edition. Australia: The Joanna Briggs Institute, 2016.

14. Higgins J, Green S. Cochrane Collaboration: Cochrane Handbook for Systematic Reviews of Interventions 
Version 5.1. 0 (updated March 2011). John Wiley/

Sons Ltd and The Cochrane Collaboration, Chichester, 2011. Available online: https://www.researchgate. net/publication/309043952_Cochrane_handbook_ for_systematic_reviews_of_interventions_Cochrane_ Collaboration

15. The National Institute for Health and Care Excellence (NICE). Hypothermia: prevention and management in adults having surgery clinical guideline (CG65) 2016. Available online: https://www.nice.org.uk/Guidance/CG65

16. Patient warming systems: Effects of patient warming methods (active vs passive) in patients undergoing major surgery. Sivapuram, Madhava Sai (MBBS Student). (Evidence Summaries) AN: JBI801 Year of Publication 2020. Available online: https://jbi.global/

17. V.Evidence Summary. Inadvertent Perioperative Hypothermia: Prevention and Management. The JBI EBP Database.2019; JBI22095. Available online: https://jbi. global/

18. Ma ZL, Yi J. Expert Consensus on Prevention and Treatment of Perioperative Hypothermia (2017). Medical Journal of Peking Union Medical College Hospital 2017;8:352-8.

19. Birch DW, Dang JT, Switzer NJ, et al. Heated insufflation with or without humidification for laparoscopic abdominal surgery. Cochrane Database Syst Rev 2016;10:CD007821.

20. Madrid E, Urrútia G, Roqué i Figuls M, et al. Active body surface warming systems for preventing complications caused by inadvertent perioperative hypothermia in adults. Cochrane Database Syst Rev 2016;4:CD009016.

21. Warttig S, Alderson P, Lewis SR, et al. Intravenous nutrients for preventing inadvertent perioperative hypothermia in adults. Cochrane Database Syst Rev 2016;11:CD009906.

22. Nieh HC, Su SF. Meta-analysis: effectiveness of forced-air warming for prevention of perioperative hypothermia in surgical patients. J Adv Nurs 2016;72:2294-314.

Cite this article as: Wang M, Jiang Y, Han W, Jiang L, Mao C. Summary of the best evidence for the prevention of intraoperative unplanned hypothermia in patients undergoing laparoscopic surgery. Gland Surg 2021;10(9):2790-2798. doi: 10.21037 /gs-21-578
23. Dean M, Ramsay R, Heriot A, et al. Warmed, humidified $\mathrm{CO} 2$ insufflation benefits intraoperative core temperature during laparoscopic surgery: A meta-analysis. Asian J Endosc Surg 2017;10:128-36.

24. Wang J, Mao XG. Intraoperative and postoperative impact of CO_2 temperature and humidity to gynecologic laparoscopic surgery: a meta-analysis. China Journal of Endoscopy 2017;23:49-56.

25. Hara K, Kuroda H, Matsuura E, et al. Underbody blankets have a higher heating effect than overbody blankets in lithotomy position endoscopic surgery under general anesthesia: a randomized trial. Surg Endosc 2021. [Epub ahead of print]. doi: 10.1007/s00464-021-08335-y.

26. Min SH, Yoon S, Yoon SH, et al. Randomised trial comparing forced-air warming to the upper or lower body to prevent hypothermia during thoracoscopic surgery in the lateral decubitus position. Br J Anaesth 2018;120:555-62.

27. Wang CQ, Hu Y. JBI evidence pre-classification and evidence rank system (2014 Edition). Journal of Nurses Training 2015;30:964-7.

28. Yi J, Xiang Z, Deng X, et al. Incidence of Inadvertent Intraoperative Hypothermia and Its Risk Factors in Patients Undergoing General Anesthesia in Beijing: A Prospective Regional Survey. PLoS One 2015;10:e0136136.

29. Yi J, Zhan L, Lei Y, et al. Establishment and Validation of a Prediction Equation to Estimate Risk of Intraoperative Hypothermia in Patients Receiving General Anesthesia. Sci Rep 2017;7:13927.

30. Su SF, Nieh HC. Efficacy of forced-air warming for preventing perioperative hypothermia and related complications in patients undergoing laparoscopic surgery: A randomized controlled trial. Int J Nurs Pract 2018;24:e12660.

(English Language Editor: C. Betlzar) 